\title{
Much Ado About the New Definitions of Sepsis
}

\author{
Sanda-Maria Copotoiu1 ${ }^{*}$, Ruxandra Copotoiu² \\ 1 University of Medicine and Pharmacy of Tirgu Mures, Romania, Clinic of Anesthesia and Intensive Care Emergency \\ County Hospital of Tîrgu Mures, Gheorghe Marinescu street no 38, Tirgu Mures, Romania \\ 2 Les Hôpitaux Universitaires de Strasbourg, Pôle d’anesthésie- réanimations chirurgicales- SAMU- SMUR, Service \\ d'anesthésiologie, Hôpital Civil
}

\begin{abstract}
Following the publication of the new definition of sepsis (Sepsis-3), a plethora of articles have been published in medical journals. Recognizing the epidemiological importance of the previous definitions, first issued in 1992 (Sepsis-1), and subsequently revised in 2001 (Sepsis-2), the most recent opinion emphasizes the failure "to provide adequate groups of patients with homogenous aetiologies, presentations and outcomes", and blamed one of the causes "for the failure of several randomized controlled trials (RCTs), that tested the efficacy of adjuvant sepsis therapies". This review summarizes the recent advances in sepsis definition.
\end{abstract}

Keywords: sepsis definition, sepsis-3

Received: 15 February 2016 / Accepted: 28 March 2016

\section{THE ISSUE}

Following the publication of the new definition of sepsis (Sepsis-3), a plethora of articles have been published in medical journals. Since to April 24 2016, over 145 583 PubMed abstracts refer to the same subject.

\section{LIVING HISTORY ON THE DEVELOPMENT OF A CONCEPT}

Recognizing the epidemiological importance of the previous definitions, first issued in 1992 (Sepsis-1), and subsequently revised in 2001 (Sepsis-2), the most recent opinion emphasizes the failure "to provide adequate groups of patients with homogenous aetiologies, presentations and outcomes", and blamed one of the causes "for the failure of several randomized controlled trials (RCTs), that tested the efficacy of adjuvant sepsis therapies" [1]. Thus a working group set the scene at the $3^{\text {rd }}$ International Consensus Definitions for Sepsis and Septic Shock (Sepsis-3) [2]. Acknowledging the problems in defining health problems, the March issue of the Intensive Care Medicine Journal enlists sepsis, along with SIRS, as one of "the ten «diseases» that are not true diseases" [1].

\section{PRECEDING VIEW POINTS ON SEPTIC SHOCK DEFINITIONS AND CRITERIA}

A Intensive Care Medicine (2015) signed by, L. Gattinoni, VM Ranieri and A. Pesenti, summarized striking differences in outcome between five recently published clinical trials: the ALBIOS, the Sepsispan, the Triss, the ProCess and the ARISE [3]. The patients in the French Sepsispan study had an expected hospital mortality of 60-62\%, while those from Australasia /New Zealand experienced a rate of 22.9-25.6\%. Common sense would dictate that differences in outcome were possibly due to different degrees of severity of the randomized patients submitted to the same treatment. The authors exemplified their point of view with the efficacy of "he prone position" in severe ARDS compared to a lack of effect in mild ARDS. Gattinoni et al used the lessons of the sepsis trials to raise awareness of the urgent need of a better classification of sepsis severity. This would avoid misleading guidelines and recommendations [3].

Critical Care (2015) published an article authored by seven intensivists. The first author, Shankar-Hari, later proved to be the initiator of the epidemiology on which as the basis of the new definitions of sepsis. The article outlined the quality judgement of the current defini-

\footnotetext{
Correspondence to: Sanda-Maria Copotoiu, University of Medicine and Pharmacy of Tirgu Mures, 38 Gheorghe Marinescu street, Tirgu Mures, 540139, Romania. E-mail: sanda.copotoiu@umftgm.ro

Ruxandra Copotoiu: Les Hôpitaux Universitaires de Strasbourg, Pôle d'anesthésie - réanimations chirurgicales - SAMU - SMUR, Service d'anesthésiologie, Hôpital Civil, Strassbourg: 1 place de l'hôpital, BP 426, 67091 Strasbourg.
} 
tions and criteria of septic shock. The feasibility, reliability and validity characteristics of the criteria were dissected. Advocating the concept of illness rather than the criteria used, they proposed that concurrent presence of cardiovascular dysfunction and perfusion/cellular abnormalities reduce the chance of improving the validity of the septic shock diagnostic. They believed this would not obstruct clinical care, but would rather aid in the design of studies aiming to identify illnessspecific biomarkers and interventions [4]. Another review article, analyzed the diagnostic criteria for sepsis and septic shock, with the emphasis on the pathophysiology [5]. Looking at the host response in severe sepsis, it appears that the proinflammatory responses, in its excess form, ultimately leads cells necrosis and damage-associated molecular patterns, known to perpetuate infections. The anti-inflammatory response is responsible for immunosuppression with enhanced susceptibility to secondary infections.

\section{THE PROFESSIONALS. INTENSIVISTS DEDI- CATED TO ELUCIDATE AN ENDANGERING MORBIDITY}

Clinical trials reporting on outcomes of severe sepsis and shock in patients submitted to EGDT, or to usual care or to some alternative resuscitation strategy were analyzed [6]. Surprisingly, patients presenting at the Emergency Department with septic shock had similar outcomes, if submitted to early "goal directed therapy" or to a non-EGDT. Moreover, EGDT was associated with increased admission to an ICU.

In order to reduce the risk of bias in the estimate of the treatment, the authors of the most recent and comparable clinical trials on the topic (the PROMISE, the ARISE and the Process) co-authored a meta-analysis of the risk of death by pooling of data [7]. In an attempt to avoid bias, writers, on behalf of the investigators of Process, ARISE and ProMISe, analyzed the methodology of the clinical trials.

They saw that harmonization of study design and methods between severe sepsis trials was feasible and might facilitate pooling of data on completion of trials. This was indeed a quality assessment exercise. All the trials conformed to the CONSORT guidelines, addressed the same fundamental questions and sheared the key-elements of design. Results were therefore comparable.
They concluded that their meta-analysis of all published RCTs on EGDT did not show improved survival for patients randomized benefitting of EGDT, compared to usual care or less invasive alternative haemodynamic resuscitation protocols. Their findings did not support the systematic use of EGDT for all patients with septic shock or its inclusion in the Surviving Sepsis Campaign (SSC). They observed that EGDT was associated with increased admission to ICU. It seemed to be a disconcerted day for those who advocated the use of EGDT.

However, since the pathogen factors (including load, virulence and pathogen-associated molecular patterns) are not modifiable, perhaps the host factors could. Environment, other illnesses and medications could be altered even if genetics resistance and age could not be [5].

\section{- THE PRODUCT OF THE EXPERTS}

In February 2015, the JAMA published the latest definitions as published in Sepsis-3 [2].

SIRS (systemic inflammatory response syndrome) is gone and seems to be buried forever, victim of an excessive focus on inflammation and of its inadequate specificity and sensitivity. The creation of the newest definitions was an expert consensus process based on the current understanding of the biopathology.

The ecologic validity was addressed utilizing multiple large electronic health record data-bases [2]. The working group issued a simplified quick SOFA score. The qSOFA criteria are:

- Respiratory rate $\geq 22 / \mathrm{min}$

- Altered mentation measured as any GCS $<15$

- Systolic blood pressure $\leq 100 \mathrm{mmHg}$

These criteria may be easily recognized even by nonphysicians and may prompt ED attendance.

Sepsis is defined as a life-threatening organ dysfunction caused by a dysregulation host response to infection.

Organ dysfunction corresponds to an acute change in total SOFA score $\geq 2$ points consequent to infection.

SOFA $\geq 2$ pts reflects overall mortality risk of approximately $10 \%$ in a general hospital population with suspected infection.

Bedside qSOFA allows for the rapid identification of those patients with suspected infection likely to have a prolonged ICU stay or to die in hospital. 
Septic shock is a subset of sepsis in which underlying circulatory and cellular/metabolic abnormalities are profound enough to substantially increase mortality.

Systolic BP was used as a criterion for qSOFA because it was most widely recorded in the electronic health record data sets.

In order to identify the patients in septic shock, the physician should first observe the clinical construct of sepsis while the blood pressure is persistently low and requiring vasopressors to maintain a MAP of at least $65 \mathrm{mmHg}$ with a serum lactate over $2 \mathrm{mmol} / \mathrm{l}(18 \mathrm{mg} \%)$ despite adequate volume resuscitation. These criteria are associated with a mortality in excess of $40 \%$.

The authors provide a flowchart that allows the practitioner to quickly diagnose sepsis or septic shock and to act accordingly [2].

For the sake of history, it should be mentioned that "septicemia", an archaic term familiar to the media and lay-men, is not completely dropped, and so interfers with the definition of sepsis [8].

The Critical Care Medicine Journal was equally interested in publishing an article that explains the process of development of interpretation of the new definitions, datailing the knowledge, the purpose and the statistical problem [9]. This favours the idea that when undertaking triage at admission to the ICU using the available biomarkers, it isnot always clear which patients are infected and which are not.

Another article on the application of a framework to assess the usefulness of alternative sepsis criteria, further explains the process of the new definitions, grading, by intended purpose, the six domains of usefulness for potential sepsis diagnostic criteria and their priority [10]. To give an example of practicality, for clinical care, the priority is high for the following domains: content validity, construct validity, criterion validity, measurement burden and timeliness. As for reliability, the prority by intended purpose is moderate-high [10].

\section{口THE REACTING STAKEHOLDERS}

Abraham wrote an editorial referring to the new definitions of sepsis and anticipated that the new definitions will be limited in their use to strengthen the design of clinical trials and, most importantly, in directing care for individual patients. His editorial remains skeptical as to the capacity of identification of patients whose organ system dysfunction is truly secondary to the underlying infection rather than other causes. This is considered to be a major limitation of the new definitions [11].

Simpson wrote that "It is a daunting undertaking to assign clinical definitions to a "condition», sepsis, which is associated with a high mortality rate, has variable clinical presentations, and has few unifying pathophysiologic features." [12].

The article dismantles Seymour's supporting paper, deemed to be a non-sequitur, ..."having used a sophisticated retrospective analysis to demonstrate that the presence of organ dysfunction, as detected by SOFA score, optimizes the combined sensitivity and specificity for life-threatening organ dysfunction.”

The endpoint of the proposed criteria is increased specificity for predicting mortality or ICU stay of $\geq 3$ days... "ideal outcomes derive from early recognition and intervention in potentially life-threatening infections"...thus "the revised criteria may lead to failure to recognize the signs of potentially lethal infection until the combination is significantly more likely to be deadly".

The SIRS concept, deemed as not helpful, being absent in 1 of 8 patients with infection and organ dysfunction, though 7 of 8 patients with infection and organ dysfunction have SIRS, making SIRS a highly sensitive indicator for organ dysfunction. Simpson's principal concern was that the new definition "de-emphasize intervention in its earlier stages of sepsis when the syndrome is actually at its most treatable" [12].

\section{DEEKING THROUGH THE PERISCOPE. PRECISION MEDICINE}

Targeted treatments are individualized treatments provided to patients based on their specific genomic and cellular alterations, have been shown to be associated with improved outcomes and enhanced clinical response in patients with cancer. Individualized treatments allow for a tailored approach for sepsis, given the heterogeneity of cellular responses associated with this condition [11]. Another consistent argument is in favor of precision medicine, but due to the heterogeneity of the cellular responses associated with sepsis, not acknowledged in the current definitions, this possibility is overruled. The new definition does not alleviate the concern that sepsis is a syndrome and not a specific disease. Incorporation of more information about the 
molecular and cellular characterization of sepsis may have been helpful.

Since cellular characterization is not a timely bedside tool, exploring the serum of the sick patients is handy and sometimes even evocative.Thus new biomarkers emerge, pushed upfront by their early detection and value. One of the most intensely studied is presepsin. A key message derived from a study of presepsin is: the pooled sensitivity and specificity of presepsin for diagnosis was 0.86 and 0.78 respectively; the area under the SROC curve was 0.89 ;presepsin has a very good diagnostic accuracy (AUC 0,89$)$ for the diagnosis for sepsis in patients with SIRS.

However, it cannot be recommended as the single definitive test for sepsis diagnosis according to current data [13]. The authors could only speculate that if added to the qSOFA criteria, presepsin could hasten the diagnosis of sepsis and thus prompt the early treatment [13].

\section{PANELS OF BIOMARKERS}

Although there is caution as to the practical value of biomarkers, serious studies add value to our practice.

Rivers used the serum (from a repository) of the patients enrolled as controls and analyzed the biomarker content and concentration. He aimed at studying the early history of the natural circulatory biomarkers active in sepsis, biomarkers retrieved from the patients at the proximal point of hospital presentation. The study presented graphs for 13 biomarkers [14]. He concluded that the circulatory biomarkers overlap, display bimodal patterns and generally peak at $3-36$ hours, diminishing over the next 72 hours. He observed that one needs to recalibrate the time of enrollment to be within the frame of the window of peak circulatory biomarker concentration [14]. From his perspective, panels of biomarkers would be the right choice in the future.

Panels come with expenses and add to the financial burden of the critically ill. Still, the chance to change the outcome of a critically ill for the better, would be money well spent.

Meanwhile, the body of evidence supports the concept of haemodynamic optimization with better intravenous fluid administration as an essential component of sepsis management [15]. Data were published showing that a positive fluid balance was an independent prognostic factor in patients with sepsis [15].

\section{SURVIVING SEPSIS CAMPAIGN TODAY}

Surviving Sepsis Campaign (SSC) developed following the ground-breaking study of E. Rivers et al, who reported an impressive effect on mortality of the patients admitted to the ED with severe sepsis. The beneficial effect was attributed to the application of a tight monitoring and therapeutic maneuvers with the intent of haemodynamic optimization [16].

SSC, by its promotors, became quite a living organism, was joined by reputed academics and practitioners, all advocating its potential life-saving approach. But as time elapsed, a growing number of studies reported disappointing results of applying EGDT versus usual care Mouncey PR. A recent study investigated 1260 patients pooled from 56 hospitals in England, half assigned to receive EGDT, half assigned to usual care and checked for all-cause mortality at 90 days. It concluded that in patients with septic shock identified early, EGDT did not improve the outcome, but led instead to increased ICU stay and costs [17].

In 2016, the Surviving Sepsis Campaign responded quickly to Sepsis-3. The Executive Committee offers clarifications and guidance for hospitals and practitioners. Their approach is also tailored to three steps, starting with the "screening and management of infection". Identification of infection comes first as the early diagnostic aim. It requires simultaneous blood and other cultures with tailored antibiotics [18]. The second step consists in the screening for organ dysfunction and management of sepsis. There is no change compared to the previous severe sepsis criteria for organ dysfunction. The qSOFA is used in this respect as a secondary screen. The patients screened positive for infection and for 2 or 3 qSOFA elements are at risk for clinical deterioration and should be closer monitored. Once organ dysfunction is acknowledged as present, the priority should be to ensure the " 3 -hour bundle" elements. The ultimate step is dedicated to the identification and the management of initial hypotension. Infected patients, be they hypotensive or with a lactate level of at least $4 \mathrm{mmol} / \mathrm{l}$, should receive $30 \mathrm{ml} / \mathrm{kg}$ crystalloids. They have to be reassessed for volume responsiveness or tissues perfusion, thus completing the six hour elements of care. The SSC promoters belief in that as it was the case for stroke and acute myocardial infarction, the early implementation of the bundles of care could make an important difference in the outcome. 


\section{A MAGNIFYING GLASS}

And here we have a late breaker, an article referring to novel risk prediction scores for community- acquired sepsis and severe sepsis that was internally validated [19]. The authors used the cohort of the REGARDS study implying 6.6 years of median observation period. They counted 1532 first sepsis with a prevalence of $8.3 \% 0$ persons-years, 1151 first severe sepsis events with a prevalence of 8.3 per 1000 person-years. They derived the SRS Model 1 and model 2 (adding biomarkers), but also SSRS severe sepsis risk score Model 1 and 2 (add biomarkers). The risk was graded as: very low, low, medium, high and very high

They concluded that the Sepsis Risk Score (SRS) and the Severe Sepsis Risk Score (SSRS) accurately predict 10 -years risk of sepsis and severe sepsis among community - dwelling adults. This tool is based upon the patients' pattern of comorbidities. They hope this might help in the prevention of sepsis and in mitigation efforts potentially useful for outpatients population [19]. The SRS and SSRS could be used to select high risk individuals most likely to benefit from novel therapies effective at sepsis prevention [19].

Adding biomarkers seems to be useful. As for the extensively promoted procalcitonin (PCT), several RCTs have shown its safety and efficacy if used to discontinue antibiotic therapy in patients with severe sepsis and shock. PCT is less convincing for treatment initiation or for withholding therapy initially. It is believed therefore that biomarkers should not be used alone, but in addition to microbiological information [20].

\section{- TO CUT THE LONG STORY OF A HOT AND LIFE THREATENING CONDITION SHORT}

I could resume by saying that the European Society of Intensive Care Medicine's and the Society of Critical Medicine's Third International Consensus Definitions for Sepsis and Septic Shock (Sepsis-3), backed up by the response of the Surviving Sepsis Campaign triggered numerous statements. Besides the academic verbalization, pushed to pedantry, with hints of refined epidemiology, it appears adequate to use simplified user-friendly scores for sepsis, in hospital or in the community, in the hope of modify the outcome by means of modifiable risk factors. There is still an under optimal knowledge and performance in the identification of infection.
To conclude, for the intensivists charged with the difficult task of altering for the best, a disappointing condition, sepsis, we face the following:

New definitions, to refine the previous ones, with the aim to help doctors and patients with a timely screening and awareness of the potential severity of the disease.

Globalization: prediction scores for sepsis and severe sepsis for the community to shift the paradigm; act upon modifiable risk factors before landing in the ICU.

Paraphrasing a former prime-minister, I could say that "scores are what they are...just scores". This follows studies comparing severity scores to septic shock patients that do not perfectly match other findings. For instance, a study carried in an academic ICU with a modern ED (Emergency Department), on a number of 56 patients admitted in the ICU with septic shock and a crude mortality of $60.71 \%$, found that SAPS score was superior for predicting survival despite the fact that the largest AUC belonged to SOFA (0.705) compared to APACHE II (0.622) and SAPS II (0.575) [21].

Still the identification, the knowledge and the performance in fighting infection is less than optimal and there is continuous elusiveness as to septic syndromes refusing to identify themselves as disease.

Of course the industry is not frozen and hits the market with scientific gadgets, such as cytokine adsorbents. The emerging technology, as part of the refinement of renal replacement therapies (RRTs), offers CytoSorb therapy consisting in removal of inflammatory mediators, mainly cytokines and chemokines. The CytoSorb adsorber removes a wide range of molecules between $5-60 \mathrm{kDa}$ molecular weight, including myoglobin, free hemoglobin, bilirubin and bile acids, but also toxins, drugs (amlodipine, venlafaxine) and substances with high protein binding. Hopes are attached to this technique, since early and some unpublished results support figures of lower mortality in septic shock attributable to CytoSorb [22].

A Cytosorb Registry is spreading its wings as we reflect on the new definitions of sepsis, as a living proof that results do not wait for definitions to adapt.

\section{DCONFLICT OF INTEREST}

Nothing to declare. 


\section{REFERENCES}

1. Depuydt PO, Kress P, Salluh JIF. The ten "diseases" that are not true diseases. Intensive Care Med. 2016; 42:411-4.

2. Singer M, Deutchman CS, Seymour CW, Shankar-Hari M, et al. The Third International Consensus Definitions for Sepsis and Septic Shock (Sepsis-3). JAMA. 2016; 315: 801-10.

3. Gattinoni L, Ranieri VM, Pesenti I. Sepsis: needs for defining severity. Intensive Care Med. 2015. 41: 551-2.

4. Shankar-Hari M, Bertolini G, Brunkhorst FM, et al. Judging quality of current septic shock definitions and criteria. Critical Care. 2015;19:445

5. Angus DC, van der Poll T. Severe sepsis and septic shock. N Engl J Med. 2013: 840-51.

6. Angus DC, Barnato AE, Bell D, Bellomo R, Choong C-R, Coats TJ et al. A systematic review and meta-analysis of early goaldirected therapy for septic shock: the Arise, ProCESS and ProMISe Investigators. Intensive Care Med. 2015;41:1549-60.

7. *** The ProCESS/ARISE/ProMISe Methodology Writing Committee. Harmonizing international trials of early goaldirected resuscitation for severe sepsis and septic shock: methodology of ProCESS, ARISE, and ProMISe. Intensive Care Med. 2013;39:1760-75.

8. Lever A, Mackenzie I. Sepsis: definition, epidemiology, and diagnosis. BMJ. 2007;335:879-83.

9. Angus DC, Seymour CW, Coopersmith CM, et al. A Framework for the Development and Interpretation of Different Sepsis Definitions and Clinical Criteria. Crit Care Med. 2016;44:e113-e121.

10. Seymour CW, Coopersmith CM, Deutchmann CS, et al. Application of a framework to Assess the Usefulness of Alternative Sepsis Criteria. Crit Care Med. 2016;44:e122-e130.
11. Edward A. Continuing evolution but with much still to be done. JAMA. 2016;315:757-9.

12. Simpson QS. New Sepsis Criteria: A Change We Should Not Make. Chest. 2016. [Epub ahead of print] doi: 10.1016/j. chest.02.653

13. Zhang $X$, Liu D, Liu Y-N, Wang R, Xie L-X. The accuracy of presepsin (sCD14-ST) for the diagnosis of sepsis in adults: a meta-analysis. Critical Care. 2015;19: 323.

14. Rivers EP, Jaehne AK, Nguyer $\mathrm{HB}$, et al. Early biomarker activity in severe sepsis and septic shock and a contemporary review of immunotherapy trials: Not a time to give up, but to give it rearlier. Shock. 2013;39:127-37.

15. Acheampong A, Vincent JL. A positive fluid balance is an independent prognostic factor in patients with sepsis. Crit Care. 2015;19:251.

16. www.sccm.org/sepsis redefined

17. Mouncey PR, Osborn TM, Power GS, et al. Trial of early, GoalDirected Resuscitation for Septic Shock. N Engl J Med. 2015; 372:1301-11.

18. www.sccmresponds to sepsis-3

19. Wang H, Donnelly JP, Griffin R, et al. Derivation of Novel Risk prediction scores for community-acquired sepsis and severe sepsis. Crit Care Med. 2016; [Epub ahead of print] doi: 10.1097/CCM.0000000000001666.

20. Albrich WC, Harbarth S. Pros and cons of using biomarkers versus clinical decisions in start and stop decisions for antibiotics in the critical care setting. Intensive Care Med. 2015;41:1739-51.

21. Georgescu A-M, Szederjesi J, Copotoiu S-M, Azamfirei L. Predicting scores correlations in patients with septic shock-a cohort study. Rom J Anaesth Int Care. 2014;21:95-8.

22. http://cytosorb-therapy.com 